\title{
LOCAL GUIDED WAVEFIELD ANALYSIS FOR CHARACTERIZATION OF DELAMINATIONS IN COMPOSITES
}

\author{
M. D. Rogge ${ }^{1}$ and C. A. C. Leckey ${ }^{1}$ \\ ${ }^{1}$ NASA Langley Research Center, Hampton, VA 23681
}

\begin{abstract}
Delaminations in composite laminates resulting from impact events may be accompanied by minimal indication of damage at the surface. As such, inspection techniques are required to ensure defects are within allowable limits. Conventional ultrasonic scanning techniques have been shown to effectively characterize the size and depth of delaminations but require physical contact with the structure. Alternatively, a noncontact scanning laser vibrometer may be used to measure guided wave propagation in the laminate structure. A local Fourier domain analysis method is presented for processing guided wavefield data to estimate spatially-dependent wavenumber values, which can be used to determine delamination depth. The technique is applied to simulated wavefields and results are analyzed to determine limitations of the technique with regards to determining defect size and depth. Finally, experimental wavefield data obtained in quasi-isotropic carbon fiber reinforced polymer (CFRP) laminates with impact damage is analyzed and wavenumber is measured to an accuracy of $8.5 \%$ in the region of shallow delaminations.
\end{abstract}

Keywords: Ultrasonic wavefield imaging, Windowed Fourier transforms, Guided waves, Structural health monitoring, Nondestructive evaluation

PACS: 43.35.Zc, 43.35.Cg, 43.60.Gk, 42.79.Qx, 43.35.Yb

\section{INTRODUCTION}

The use of composites in commercial airframes is dramatically increasing. With the increased use of composites, different forms of damage are expected to occur throughout the life of the vehicle. Of particular concern is impact damage caused by hail, tool drops, or collision with ground service vehicles. Inspection standards typically rely on visual inspection for damage detection and subsequent ultrasonic characterization. Structural health monitoring aims to improve damage detection through automated interrogation of the structure. Application of guided waves towards damage detection, localization, and measurement is an active research area. Structural health monitoring systems commonly 
utilize permanently bonded piezoelectric transducers to transmit and receive guided waves and analyze the received signals using a variety of algorithms to detect, localize, and characterize damage. However, the fidelity of characterization is limited when compared to conventional ultrasonic inspection techniques where damage sizing can be accomplished to better than $1 \mathrm{~mm}$ resolution [1]. In addition, conventional scanning systems require significant operator skill and setup time [2]. Researchers have recently been investigating non-contact guided wavefield imaging methods utilizing air-coupled and laser Doppler vibrometer based measurements in combination with permanently attached piezoelectric sensors as an alternative to traditional contact or scanned ultrasonic methods $[3,4]$. Such methods are able to interrogate a region of the structure and generate a large amount of data containing quantitative damage information. In order to process the large datasets produced by these techniques, data analysis methods have been developed that provide information related to presence of damage through incident wave removal [5], standing wave filtering [6], frequency-wavenumber analysis [7], spatial spectrograms [8], and multi-dimensional continuous wavelet transforms [9]. This paper presents a windowed Fourier transform-based analysis of wavefield data that provides a measurement of principal wavenumber at each measurement location. The method is applied to both simulation data and to experimentally obtained guided wavefields that contain interactions with delaminations in composite laminates.

\section{WAVEFIELD ANALYSIS METHOD}

\section{Mode filtering}

In general, ultrasonic transducers will generate multiple propagating guided wave modes in plate like structures. The wavenumber spectra of the wavefield will therefore contain peaks corresponding to the various modes. In order to reduce the effects of any modes other than the mode of interest, the entire wavefield can be filtered in the wavenumber domain [10]. This is accomplished by applying a three-dimensional filter to the wavefield that passes the interrogating mode and rejects all others within the frequency bandwidth of the excitation signal. Such a process is shown in Eq. 1:

$$
\tilde{U}\left(k_{x}, k_{y}, \omega\right)=W_{k}\left(k_{x}, k_{y}, \omega\right) U\left(k_{x}, k_{y}, \omega\right)
$$

where $U\left(k_{x}, k_{y}, \omega\right)$ is the three-dimensional Fourier transform of the wavefield $u(x, y, t)$. In this work, the filter $W_{k}\left(k_{x}, k_{y}, \omega\right)$ is an axisymmetric ideal band-pass filter centered about the nominal wavenumber of the mode of interest and defined by low and high wavenumber cutoffs $k_{l}$ and $k_{2}$, respectively as shown below.

$$
W_{k}\left(k_{x}, k_{y}, \omega\right)= \begin{cases}0, & |\mathbf{k}|<k_{1} \\ 1, & k_{1} \leq|\mathbf{k}| \leq k_{2} \\ 0, & |\mathbf{k}|>k_{2}\end{cases}
$$

where the wavenumber vector $\mathbf{k}$ is

$$
\mathbf{k}=\left[\begin{array}{l}
k_{x} \\
k_{y}
\end{array}\right]
$$

The filtered wavefield is then transformed back into the spatio-temporal domain via the inverse three-dimensional Fourier transform

$$
\tilde{u}(x, y, t)=\mathcal{F}_{3 D}^{-1}\left(\tilde{U}\left(k_{x}, k_{y}, \omega\right)\right)
$$


to provide the filtered wavefield $\tilde{u}(x, y, t)$.

\section{Spatial windowing}

With the wavefield containing only a single mode, a grid of $m$ by $n$ measurement locations corresponding to $x=x_{m}$ and $y=y_{n}$ are defined. The filtered dataset is then multiplied by a spatial window to produce the windowed dataset $\tilde{u}_{m n}$

$$
\tilde{u}_{m n}(x, y, t)=w_{x y}(x, y) \tilde{u}(x, y, t)
$$

where in this work the window $w_{x y}$ is a axisymmetric Hann window of diameter $D$

$$
w_{x y}=0.5\left(1-\cos \left(2 \pi \frac{r}{D}\right)\right), 0 \leq r \leq D / 2
$$

and $r$ is defined as distance from the measurement location.

$$
r=\sqrt{\left(x-x_{m}\right)^{2}+\left(y-y_{n}\right)^{2}}
$$

\section{Principal wavenumber calculation}

Next, it is assumed that within the windowed dataset, the guided waves propagate with a single wavenumber. To measure the wavenumber within the region, the wavenumber spectrum is analyzed. This is performed by applying the three dimensional Fourier transform to the windowed dataset and selecting the two-dimensional wavenumber spectrum at the center frequency of the excitation signal, $\omega_{0}$. The wavenumber spectrum for each measurement is processed as follows. The principal wavenumber, $k_{m n}$, at location $x=x_{m}$ and $y=y_{n}$ and excitation angular frequency $\omega=\omega_{0}$ is calculated via a weighted sum over the entire wavenumber range as described in Eq. 8.

$$
k_{m n}=\frac{\sum_{\mathbf{k}} \tilde{U}_{m n}\left(k_{x}, k_{y}, \omega_{0}\right)|\mathbf{k}|}{\sum_{\mathbf{k}} \tilde{U}_{m n}\left(k_{x}, k_{y}, \omega_{0}\right)}
$$

\section{NUMERICAL AND EXPERIMENTAL METHODS}

\section{Diffusion Disbond Simulation}

Finite element simulations were performed using COMSOL to study the local Fourier analysis described above. The simulation includes a 100 by 50 by $2 \mathrm{~mm}$ thick 6061 alloy aluminum plate with a cylindrical void located within the plate to simulate a diffusion disbond as shown in Figure 1. The void is $20 \mathrm{~mm}$ in diameter and 50 microns thick. A $9.6 \mathrm{~mm}$ diameter, $0.8 \mathrm{~mm}$ thick piezoelectric disk is also included to act as a source of guided waves. The piezoelectric disk is excited by a $100 \mathrm{~V}_{\mathrm{pp}}, 200 \mathrm{kHz}$, fivecycle Hann windowed sinusoidal voltage boundary condition applied to the planar surfaces of the disc. The simulation was performed using a fixed time step solver with $250 \mathrm{~ns}$ time step and $0.2 \mathrm{~mm}$ maximum quadratic element size in order to satisfy suggested temporal and spatial steps as outlined in [11] and [12]. 

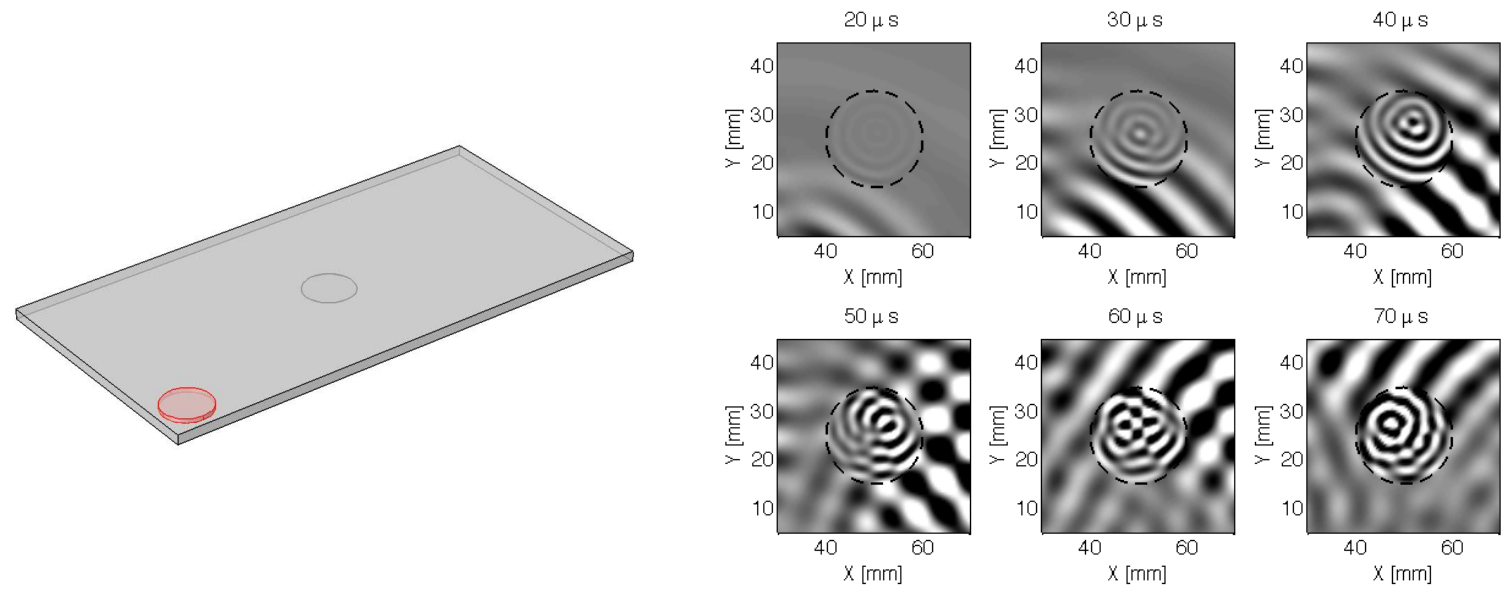

FIGURE 1. Simulation geometry (left) and calculated wavefield (right) interacting with a $20 \mathrm{~mm}$ diameter diffusion disbond located $0.2 \mathrm{~mm}$ below the plate surface. The dashed line indicates the edge of the disbond. Low amplitude $\mathrm{S} 0$ and higher amplitude A0 modes are present in the wavefield and undergo multiple transits within the region above the disbond. Wavenumber of the A0 mode is shown to increase dramatically in the presence of the disbond.

\section{Carbon Fiber Reinforced Polymer Laminate}

The experimental sample is a $3.2 \mathrm{~mm}$ thick, 26-ply carbon fiber reinforced polymer (CFRP) laminate plate with layup $\left[(0 /+45 /-45 / 90)_{3} 0\right]_{S}$. Delaminations were created in the plate through quasi-static indentation and then grown through repeated indentation. This technique is commonly used to grow delaminations in composite materials in a controlled manner. Figure 2 shows immersion time of flight $\mathrm{C}$-scans of the damage in the initial and grown states obtained with a $20 \mathrm{MHz}, 2$-inch focal length focused transducer. Initial damage is approximately $21 \mathrm{~mm}$ in diameter and was grown to approximately $50 \mathrm{~mm}$ in diameter. Many delaminations are present at various ply interfaces as indicated by the labels.

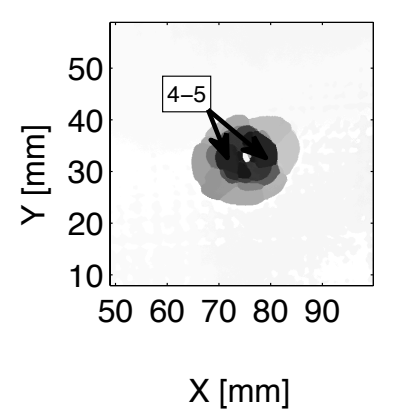

(a)

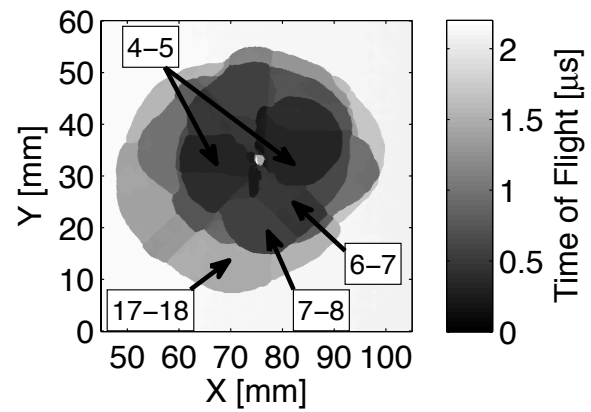

(b)

FIGURE 2. Time of flight c-scans for (a) initial and (b) grown damage. Delaminations are apparent at various depths and of increasing size with depth as is typical for impact damaged composite laminates. Labels indicate ply interfaces at which selected delaminations occur (e.g. 4-5 indicates delamination at the interface between the fourth and fifth plies).

Wavefield data was recorded in the CFRP coupon after damage was created and then again after growing the delaminations. The response at a Cartesian grid of coordinates was measured using a Polytec OFV-505 laser Doppler vibrometer attached to a two axis scanning system. The grid measures 128 by $63 \mathrm{~mm}$ with $0.5 \mathrm{~mm}$ spacing. For both damage 
states, a Panametrics V103 transducer was coupled to the plate with gel couplant and a 100 $\mathrm{V}_{\mathrm{pp}}$ chirp with bandwidth $20 \mathrm{kHz}$ to $1.12 \mathrm{MHz}$ was applied to the transducer. The measured signals were then filtered following the procedure outlined in [13] to produce the response due to a five-cycle $500 \mathrm{kHz}$ Hann windowed sinusoid. Snapshots of the wavefields with initial and grown damage are shown in Figures 3 and 4. Multiple guided wave modes and significant scattering from the plate edges and delaminations are apparent. In addition, change in wavelength is clearly evident within the grown damaged region at $t=30 \mu \mathrm{s}$.
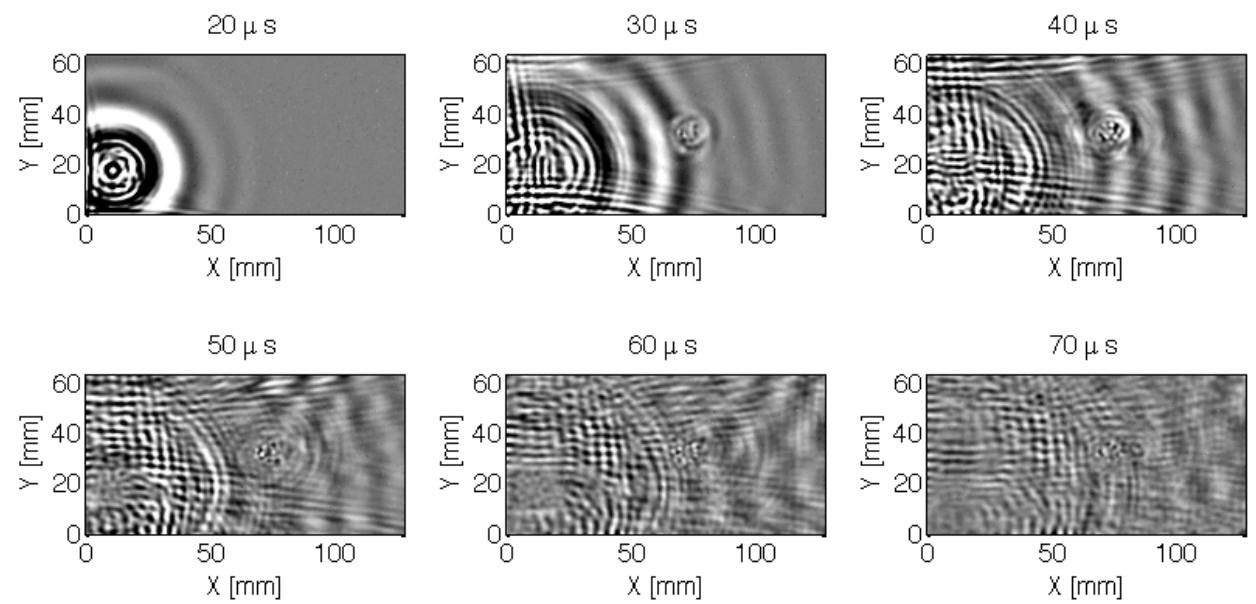

FIGURE 3. Recorded wavefield at surface of CFRP coupon with initial damage for $500 \mathrm{kHz}$ excitation frequency. Multiple modes and edge scattering are present. Wavefronts reach the damage around $t=20 \mu \mathrm{s}$ after which scattering is clearly evident.
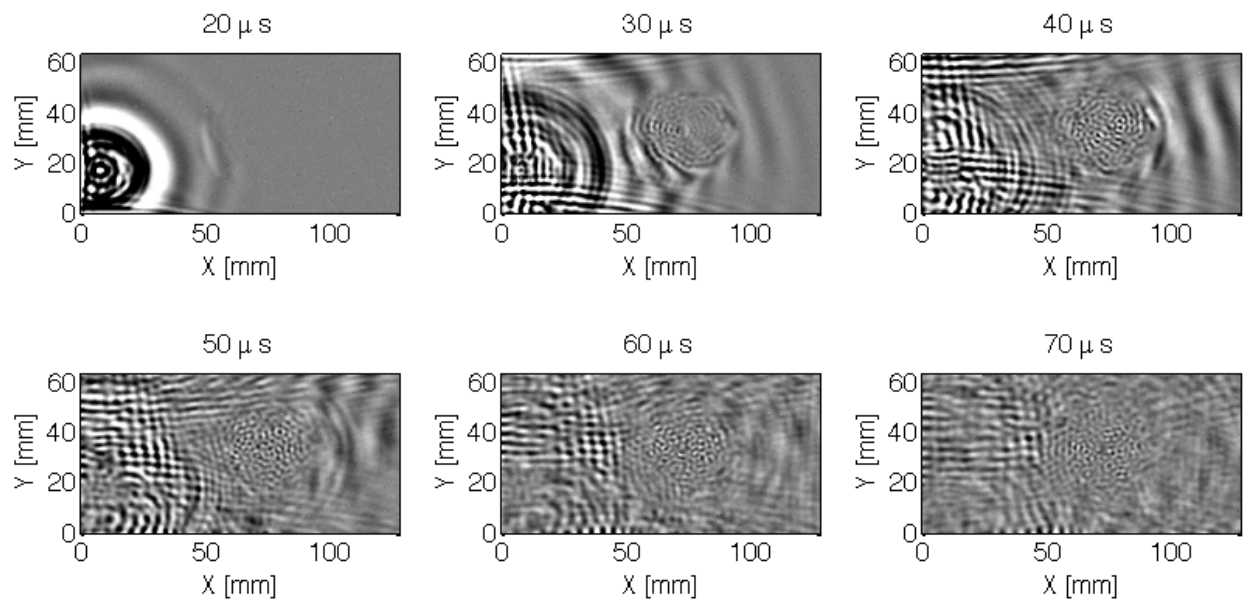

FIGURE 4. Recorded wavefield at surface of CFRP coupon with grown damage for $500 \mathrm{kHz}$ excitation frequency. Clear increase in wavenumber is apparent at the various delaminations at $t=30 \mu \mathrm{s}$.

\section{RESULTS}

\section{Simulation}

The principal wavenumber was calculated for the simulated wavefields. Figure 5 shows theoretical and measured wavenumber versus disbond depth and also versus location for a $0.2 \mathrm{~mm}$ depth disbond. There is good agreement with solutions of the antisymmetric Rayleigh-Lamb equation (dashed line) when disbond depth is less than $0.6 \mathrm{~mm}$ 
where theoretical wavenumber is $192 \mathrm{~m}^{-1}$ and measured wavenumber is $194 \mathrm{~m}^{-1}$, or $1.3 \%$ error. At this depth, wavelength is approximately $5 \mathrm{~mm}$, or half the window size. As disbond depth increases, wavelength will increase, resulting in fewer wavelengths within the spatial window leading to increased error, as evident in the figure. This is consistent with results of one-dimensional tone analysis using the short time Fourier transform where it was found that window size should be greater than two periods for accurate measurements $[14,15]$.

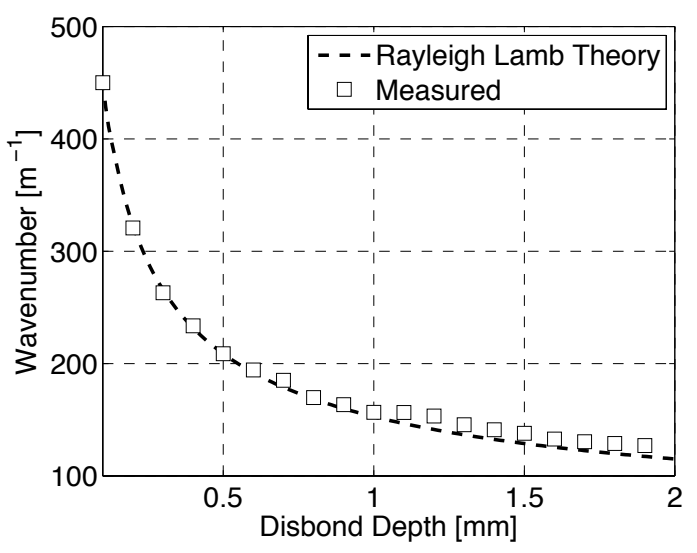

(a)

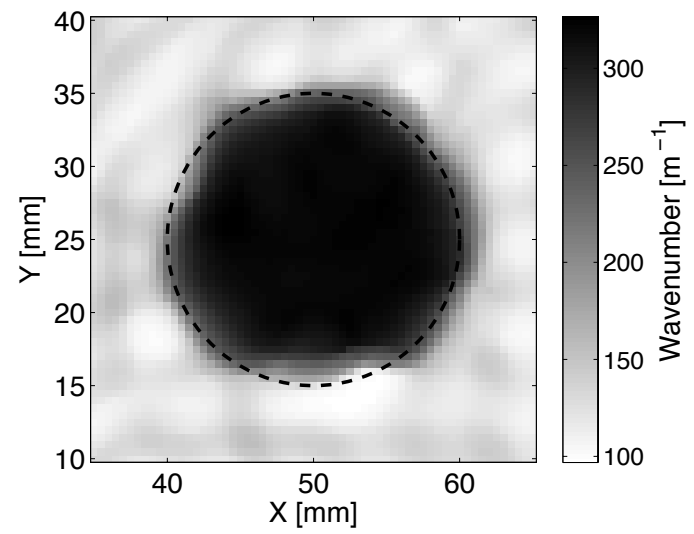

(b)

FIGURE 5. (a) Measured and theoretical wavenumber for guided waves propagating above diffusion disbonds of varying depth. (b) Measured wavenumber versus location for $0.2 \mathrm{~mm}$ depth. In both cases disbond diameter is 20 $\mathrm{mm}$ and window diameter is $10 \mathrm{~mm}$.

\section{$\underline{\text { Experiment }}$}

Experimentally measured wavefields were processed using the local Fourier analysis method outlined above. In order to determine window size, the software package DISPERSE v2.0.16i [16] was used to calculate theoretical wavenumber for a number of ply "stackups" corresponding to laminate regions above delaminations at the 4-5, 6-7, 7-8, $17-18$ ply interfaces and for the pristine 26 ply plate. Material properties used to perform the calculations are obtained from the literature and are presented in Table $1[17,18]$. The expected range of wavenumber within the coupon is determined from the two extreme cases: 1) the full 26-ply plate and 2) the top four plies [0/+45/-45/90] corresponding to the region above the delaminations at the 4-5 ply interface. At frequency equal to $500 \mathrm{kHz}$, calculated wavenumber ranges from 324 to $477 \mathrm{~m}^{-1}$. Based on this, the pass band of the mode isolation filter was determined to be 250 to $590 \mathrm{~m}^{-1}$. Given that the longest wavelength in the filtered wavefields is $4 \mathrm{~mm}$, window size was determined to be $8 \mathrm{~mm}$ in order ensure multiple wavelengths are contained within the windowed wavefields.

TABLE 1. IM7/8552 Material properties

\begin{tabular}{cccccccccc}
\hline \hline$\rho\left[\mathrm{kg} / \mathrm{m}^{3}\right]$ & $\mathrm{E}_{1}[\mathrm{GPa}]$ & $\mathrm{E}_{2}[\mathrm{GPa}]$ & $\mathrm{E}_{3}[\mathrm{GPa}]$ & $\mathrm{G}_{12}[\mathrm{GPa}]$ & $\mathrm{G}_{13}[\mathrm{GPa}]$ & $\mathrm{G}_{23}[\mathrm{GPa}]$ & $v_{12}$ & $v_{13}$ & $v_{23}$ \\
\hline 1570 & 171.4 & 9.08 & 9.08 & 5.29 & 5.29 & 2.80 & 0.32 & 0.32 & 0.5 \\
\hline \hline
\end{tabular}

Figure 6 shows the measured wavenumber versus location for the initial and grown damage cases using the $8 \mathrm{~mm}$ window. Black lines indicate delamination edges from the immersion C-scans. Variation in measured wavenumber is present in the pristine areas of the plate with an average measurement equal to $320 \mathrm{~m}^{-1}$. This variation can be attributed to the windowing process as even with multiple wavelengths within the window, small variations due to the windowing process occur. Measured wavenumber is $340 \mathrm{~m}^{-1}$ in the 
region above delaminations between plies 4 and 5 (4-5 ply interface). This is only a slight increase over wavenumber measured in the pristine plate and corresponds to a rather large error of $-25.6 \%$ from the theoretical $457 \mathrm{~m}^{-1}$.

In contrast, the measured wavenumber within the grown damage agrees much better with theoretical predictions. In the region above the 4-5 ply delaminations, average measured wavenumber is $418 \mathrm{~m}^{-1}$, an $-8.5 \%$ error relative to theoretical prediction and a marked improvement over the initial damage measurement. Table 2 presents wavenumber and percent error measured in the regions of selected delaminations. Theoretical wavenumber is given as a range due to the directional dependence resulting from anisotropic material properties of the laminate. Maximum percent error is $-12.6 \%$ for the 17-18 ply interface delamination. Within this region, measured wavenumber is actually lower than that measured in the undamaged region. As expected, as delamination depth increases, the change in wavenumber dramatically decreases. This implies the current analysis method may only be applicable for shallow delamination depths.
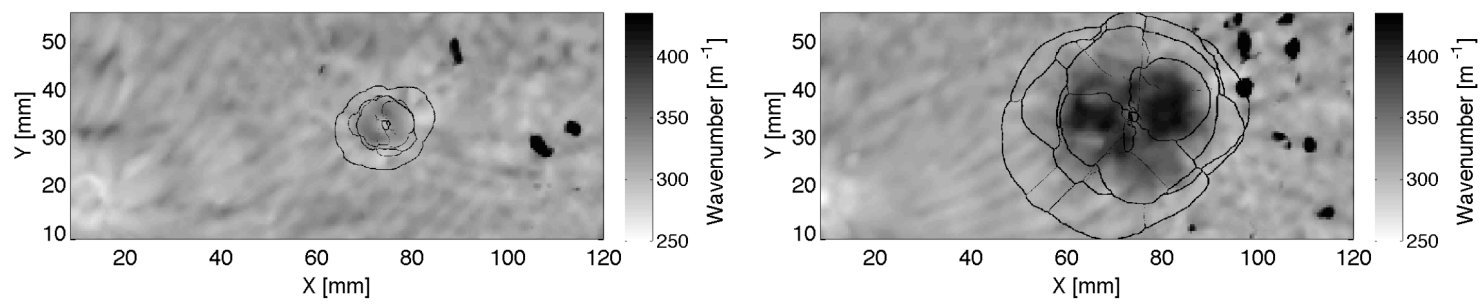

FIGURE 6. Measured wavenumber for the initial damage (left) and grown damage (right). Delamination edges obtained from immersion $\mathrm{C}$-scans are shown as black lines. The effect of delamination size on measured wavenumber is clearly evident from the improved correlation to theoretical prediction in the grown damage case. Artifacts shown in the right portions of the images are due to reduced SNR.

TABLE 2. Measured wavenumber corresponding to delaminations at various ply interfaces

\begin{tabular}{cccc} 
& \multicolumn{3}{c}{ Wavenumber $\left[\mathrm{m}^{-1}\right]$} \\
\hline \hline Ply Interface & Theory & Measured & \% Error \\
\hline $4-5$ & $437-477$ & 418 & -8.5 \\
$6-7$ & $375-444$ & 361 & -11.8 \\
$7-8$ & $360-406$ & 350 & -8.6 \\
$17-18$ & $341-371$ & 311 & -12.6 \\
Pristine Plate & $324-346$ & 321 & -4.2 \\
\hline \hline
\end{tabular}

\section{SUMMARY AND CONCLUSIONS}

We have presented a new wavefield analysis method capable of characterizing delaminations in composite laminates through local wavenumber measurements. The process was applied to both simulated and experimentally measured wavefields. Based on the simulation results, it is evident that the size of the region used to make the local measurement influences accuracy. It has been shown that similarly to previous onedimensional windowed Fourier analysis, the window size should contain multiple wave periods in order to reduce error.

The experimental measurements show good agreement with theory provided that the size of geometric features is larger than the window size. The method was unable to effectively measure wavenumber within damage that is a fraction of the window size. While the method is limited in capability for small damage, it has been shown to be 
effective in application to larger sized damage. Future work will investigate methods to reduce window size while maintaining wavenumber resolution and incorporate directionality into the measurement. Overall, the presented analysis of two dimensional wavefield data provides quantitative measurements of the wavenumber irrespective of propagation direction. The method has been shown to be effective in characterizing near surface delaminations in composite laminates.

\section{REFERENCES}

1. J.-K. Kim, "Recent developments in impact damage assessment of fibre composites," in Impact Behaviour of Fibre-Reinforced Composite Materials and Structures, edited by S. R. Reid and G. Zhou, CRC Press, Boca Raton, 2000, pp. 33-74.

2. G. Georgeson, NDE \& Health Monit. of Aerosp. Mater. Civ. Infrastruct. 4704 (2002) 104-115.

3. T. E. Michaels and J. E. Michaels, "Application of acoustic wavefield imaging to noncontact ultrasonic inspection of bonded components," in Rev. Prog. Quant. Nondestr. Eval., edited by D. O. Thompson and D. E. Chimenti 25 (2006) 1484-1491.

4. J.-R. Lee, J. Takatsubo, N. Toyama, and D.-H. Kang, Meas. Sci. Technol. 18 (12) (2007) 3816-3824.

5. T. E. Michaels and J. E. Michaels, "Monitoring and characterizing corrosion in aluminum using Lamb waves and attached sensors," in Health Monit. Struct. Biol. Syst., edited by T. Kundu 6532 (2007) 65321G-1-11.

6. H. Sohn, D. Dutta, J. Y. Yang, M. DeSimio, S. Olson, and E. Swenson, Smart Mater. Struct. 20 (4) (2011) 045017.

7. T. E. Michaels, M. Ruzzene, and J. E. Michaels, "Frequency-wavenumber domain methods for analysis of incident and scattered guided wave fields," in Health Monit. Struct. Biol. Syst. 7295 (2009) 729513-1-12.

8. C. A. C. Leckey, M. D. Rogge, C. A. Miller, and M. K. Hinders, Ultrasonics 52 (2) (2012) 193-207.

9. M. D. Rogge and P. H. Johnston, "Wavenumber imaging for damage detection and measurement," in Rev. Prog. Quant. Nondestr. Eval., edited by D. O. Thompson and D. E. Chimenti 31 (2012) 761-768.

10. M. Ruzzene, "Frequency-wavenumber domain filtering for improved damage visualization," in Smart Mater. Struct. 16 (6) (2007) 2116-2129.

11. D. N. Alleyne and P. Cawley, IEEE Trans. on Ultrason., Ferroelectr., and Freq. Control. 39 (3) (1992) 381-97.

12. F. Moser, L. J. Jacobs, and J. Qu, Nondestr. Test. Eval. Int. 32 (4) (1999) 225-234.

13. J. E. Michaels, S. J. Lee, A. J. Croxford, and P. D. Wilcox, Ultrasonics (In Press) (2012).

14. P. Depalle and T. Helie, Proc. 1997 Workshop Appl. Signal Process. Audio Acoust. (6) 4.

15. R. J. McAulay and T. F. Quatieri, IEEE Trans. Acoust. Speech Signal Proces. ASSP-34 (4) (1986) 744-754.

16. B. N. Pavlakovic, M. J. S. Lowe, D. N. Alleyne, and P. Cawley, "Disperse: a general purpose program for creating dispersion curves," in Rev. Prog. Quant. Nondestr. Eval., edited by D. O. Thompson and D. E. Chimenti 16 (1997) 185-192.

17. P. P. Camanho, P. Maimí, and C. G. Dávila, Compos. Sci. Technol. 67 (13) (2007) 27152727.

18. A. S. Kaddour, M. J. Hinton, S. Li, and P. Smith, Compos. Sci. Technol. (2008). 